\title{
Time-Resolved Laue Diffraction of Deforming Micropillars
}

\author{
Robert Maaß, ${ }^{1}$ Steven Van Petegem, ${ }^{1}$ Helena Van Swygenhoven, ${ }^{1, *}$ Peter M. Derlet, ${ }^{1}$ \\ Cynthia A. Volkert, ${ }^{2}$ and Daniel Grolimund ${ }^{1}$ \\ ${ }^{1}$ Paul Scherrer Institut, CH-5232 Villigen PSI, Switzerland \\ ${ }^{2}$ Institut für Materialforschung II, Forschungszentrum Karlsruhe, D-76021 Karlsruhe, Germany
}

(Received 10 April 2007; published 3 October 2007)

\begin{abstract}
We demonstrate real-time resolved white beam Laue diffraction during compression of micron-sized focused ion beam milled single crystals Au pillars, revealing the dynamical correlation between microstructure and plasticity. The evolution of the Laue patterns of the Au pillars demonstrates the occurrence of crystal rotation and strengthening is explained by plasticity starting on a slip system that is geometrically not predicted but selected because of the character of the preexisting strain gradient.
\end{abstract}

DOI: 10.1103/PhysRevLett.99.145505

PACS numbers: 62.20. $-\mathrm{x}, 61.72 . \mathrm{Dd}$

It is the ease of dislocation nucleation and propagation, and the nature of the dislocation multiplication mechanism, that will determine the strength of the metallic structure; a concept offering several pathways for strengthening. A well-known strengthening mechanism is the introduction of interfaces representing obstacles for dislocation propagation as is done in polycrystalline aggregates [1,2] or in dislocation pileups against a surface layer [3]. Strength can also be increased by reducing the availability of dislocation sources as is observed in small micron-sized whiskers with a perfect defect free crystalline structure [4]. Plastic deformation is then characterized by a very high initial flow stress explained by the lack of available dislocation sources, followed by an enormous strain softening due to the sudden high density of dislocations. Strengthening has also been observed when reducing object dimensions to the micron and submicron scale [512], hereafter referred to as "smaller is stronger." For example, in the compression of single crystal micron-sized pillars synthesized by focused ion beam (FIB) milling, a drastic increase in yield stress with decreasing pillar diameter has been reported [5-12]. Plastic deformation is here characterized by individual slip events that are reflected in discrete strain bursts during which the flow stress does not fall to lower values. This type of strengthening has been ascribed to a "starvation" of mobile dislocations in Refs. [5,9,10].

In past work, uniaxial compression of micron-sized pillars was performed using a nanoindenter outfitted with a flat-punch indentation tip, a setup specifically developed to study the "smaller is stronger" trend [5]. Such microcompression testing is performed on pillars that are usually machined from a bulk material using a FIB. In this geometry one end of the pillar remains part of the bulk material, while the other is a free surface. Assuming low friction contact conditions between the indenter and pillar, the compression axis is expected to rotate unconstrained with minimal crystal rotation $[7,13,14]$. Inspection of the resulting stress-strain curves reveals discrete strain bursts, and postmortem surface investigations reveal a large scattering among data with pillars of similar orientation deforming according to different slip systems, sometimes shearing off or barrelling $[7,10]$.

In this Letter we demonstrate that real-time resolved white beam Laue microdiffraction offers to go well beyond the current measurements, providing the detailed dynamics of the underlying deformation mechanism and revealing a different view on the "smaller is stronger" paradigm measured in FIB milled pillars. White beam Laue diffraction patterns are sensitive to microstructure [15-18], where continuous streaking of Laue diffraction peaks can be related to internal strain gradients and discontinuous streaking to the presence of dislocation walls forming geometrically necessary boundaries $[17,18]$. The details of the in situ microcompression device and Au pillar sample preparation are given in section A1 of the auxiliary material [19]. In situ compression of the smaller Au pillars

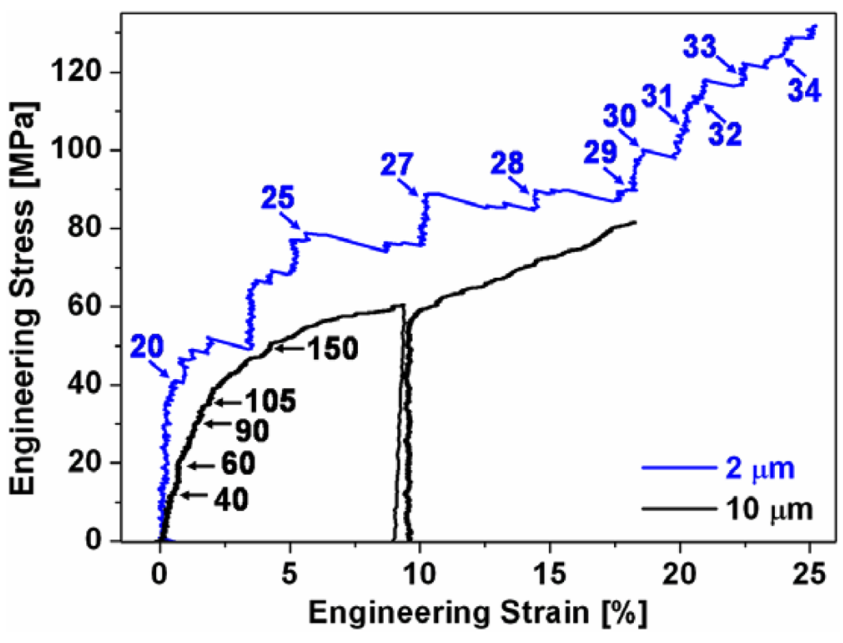

FIG. 1 (color online). The engineering stress-strain curves shown are the curves for a $2 \mu \mathrm{m}$ and a $10 \mu \mathrm{m}$ pillar. The arrows indicate the corresponding Laue patterns taken during compression. 
demonstrates how an ensemble of dislocations selforganizes prior to a strain burst, choosing a geometrically hard slip system predefined by the initial strain gradient present in the undeformed pillar. In larger pillars, where the yield strength is comparable to bulk Au, the influence of the initial strain gradient is less and slip starts on the geometrically predicted slip system. Furthermore, in contrast to what was assumed in previous work, plastic deformation is accompanied by crystal rotation for both pillar diameters.

Figure 1 shows the stress-strain curves of a 2 and $10 \mu \mathrm{m}$ $\mathrm{Au}$ pillar that have been deformed while following the Laue patterns. The engineering stress-strain values are calculated as suggested in Ref. [11] and the numbers indicated on the curve give the corresponding Laue pattern. The 2 and $10 \mu \mathrm{m}$ Au pillars have the same initial crystallographic orientation, with the compression axis being $\sim[4 \overline{6} 3]$ and incoming beam direction $\sim[134]$. The smallest pillar is deformed until $25 \%$ strain and the deformation curve is characterized by an initial elastic part followed by rapid strain bursts. Postmortem surface investigation shows slip traces according to the ( $1 \overline{1} 1)$, the $(1 \overline{1} \overline{1})$, and the $(\overline{1} \overline{1} 1)$ slip planes [Fig. 2(a)]. The stress-strain curve of the $10 \mu \mathrm{m}$ pillar, once unloaded and sequentially reloaded, contains several small strain bursts and postmortem surface investigation shows $(1 \overline{1} \overline{1})$ slip lines that are uniformly distributed all over the pillar and some (1111) slip lines at one side of the base of the pillar [Fig. 2(b)].

The white beam Laue transmission diffraction pattern recorded prior to deformation contains five diffraction spots of sufficient intensity corresponding to the reflections $(\overline{2} 00),(02 \overline{2}),(\overline{3} 1 \overline{1}),(\overline{1} \overline{3} 1)$, and $(\overline{2} 2 \overline{2})$. All Laue spots of the undeformed pillars show continuous streaking indicating the presence of a strain gradient which for $\mathrm{Si}$ has been ascribed to the FIB synthesis procedure [20]. Upon compression of the $2 \mu \mathrm{m}$ pillar all Laue spots evidence additional continuous streaking until just before the first strain burst is observed, corresponding to number 20 on the stress-strain curve. During the first strain bursts the peak sharpens, and then a satellite peak is formed, which is first visible at number 25 on the stress-strain curve. Upon a

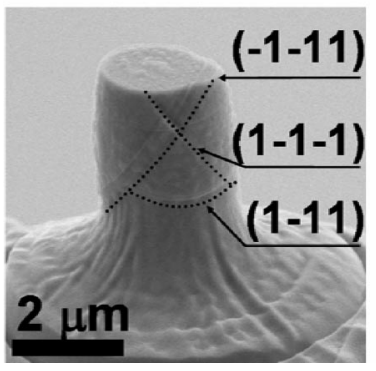

b

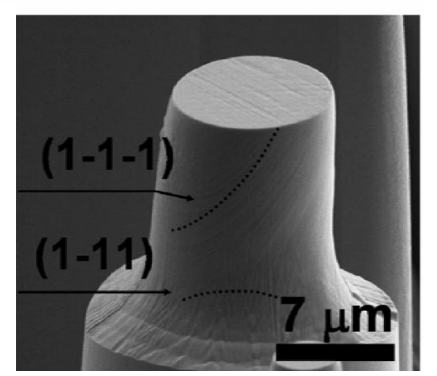

FIG. 2. Scanning electron microscopy images of (a) the $2 \mu \mathrm{m}$ pillar evidencing three active slip planes and (b) the $10 \mu \mathrm{m}$ pillar evidencing two active slip systems. further loading, this satellite peak moves in discrete steps along different directions further away from the mother peak. Such satellite behavior is visible in all peaks. Movies of the $(\overline{2} 2 \overline{2})$ and the $(02 \overline{2})$ reflections are provided in movies A-1 and A-2 of the auxiliary material [19].

The paths of all satellite diffraction peaks during deformation uniquely define a rotation of the crystal. Figure 3(a) shows, using an inverse pole plot of the primary triangle formed by the [010], [110], and the [1111] poles, the evolution of the crystal rotation by plotting the vertical axis in the reciprocal space of the crystal [21]. The geometrically predicted slip systems are shown for the primary and neighboring triangles. For compression geometries where the crystal is free to rotate, the vertical axis should rotate towards the line $[0 \overline{1} 0]-[1 \overline{1} 0]$ using the $(1 \overline{1} \overline{1})[01 \overline{1}]$ slip system, whereupon the $(1 \overline{1} 1)[0 \overline{1} \overline{1}]$ system is equally activated maintaining the orientation along the [010]-[1 10$]$ line. However, for the smallest pillar, the crystal rotates in the opposite direction, continuing towards the line

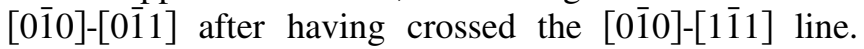
During rotation the geometrically resolved shear stress

a
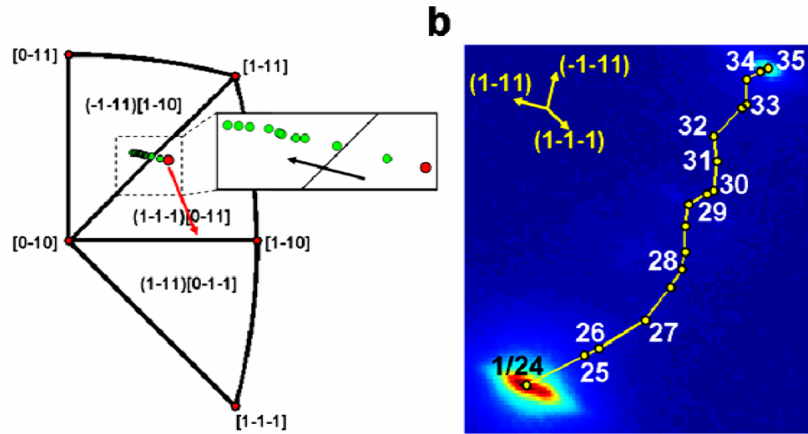

C

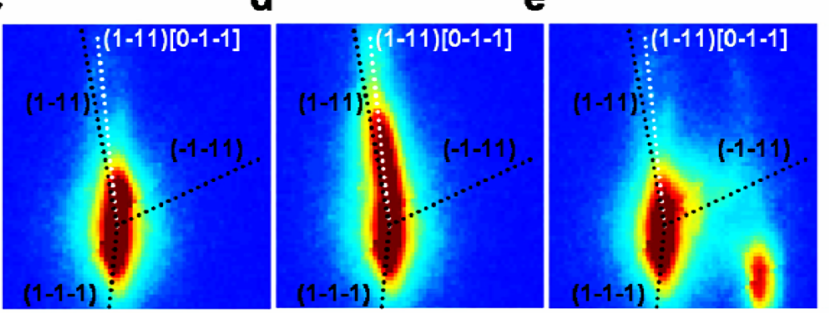

FIG. 3 (color online). Results obtained from the Laue patterns taken during deformation of the $2 \mu \mathrm{m}$ pillar. (a) Inverse pole figure showing crystal rotation via the evolution of the compression axis in the reciprocal space of the pillar. The orientation before deformation is indicated in red (gray) and the red (gray) arrow indicates the classical rotation direction. The actual path direction is given by the arrow in the inset. (b) Satellite peak path emerging from the $(\overline{2} 00)$ peak during deformation with vectors showing the different directions associated with classical rotation. The indicated numbers correspond with those on the stressstrain curve in Fig. 1. Intensity distribution of the $(\overline{2} 2 \overline{2})$ reflection prior to deformation (c), loaded to $40 \mathrm{MPa}$ (d) and loaded to $77 \mathrm{MPa}(\mathrm{e})$. White lines represent the streaking directions and black lines the peak path associated with crystal rotation. 
changes, as is determined by the Schmid factors [22] shown in Fig. A-2 of the auxiliary material [19], which displays the time evolution of the slip systems of the five highest Schmid factors.

Figure 3(b) shows the initial intensity distribution of the (200) Laue spot (Laue pattern 1), and the final position of (200) satellite (Laue pattern 35) positioned in one plot together with the intermediate path indicated by numbers on the stress-strain curve. Once the satellite has taken off, different directions can be distinguished in the movement of the peak. Because of the integration time of the detector used and the non-negligible readout time (see Ref. [19]) it is not always possible to uniquely relate the change in the direction of the Laue spot to a change in slip plane. However, it is clear that the bursts between 28-29 and $30-32$ can be assigned to the ( $\overline{1} \overline{1} 1)[1 \overline{1} 0]$ slip system, while the $29-30$ and $32-33$ bursts clearly evidence the intermittent activity of the $(1 \overline{1} \overline{1})[0 \overline{1} 1]$ system.

Figure 3(c) shows the intensity distribution of the $(\overline{2} 2 \overline{2})$ reflection prior to deformation, Fig. 3(d) at $40 \mathrm{MPa}$ when maximum streaking is achieved (corresponding to point 20 on the deformation curve), and Fig. 3(e) at $77 \mathrm{MPa}$ when the satellite is formed (Laue pattern 25). On these plots, the streaking (white lines) directions are shown for particular slip systems. Following Barabash et al. [18], the streaking direction for a particular slip system is calculated under the assumption of an excess population of pure edge dislocations and given by the intersection of the planes perpendicular to the dislocation line and the reciprocal lattice vector of the corresponding reflection. Also shown (in black) are the directions of peak movement associated with classical crystal rotation around a direction perpendicular to the load axis and the normal to the slip plane. The observed streaking in all indexed peaks can be assigned to the presence of unpaired dislocations with a Burgers vector $[0 \overline{1} \overline{1}]$ lying in the (1 $\overline{1} 1)$ slip plane. At the moment of the burst (Laue pattern 20) streaking of the Laue spot reduces. In other words, upon loading an ensemble of dislocations is formed that will self-organize to a critical slip process visible by a strain burst, bridging the gap between individual dislocations and an ensemble of dislocations preparing for macroscopic plasticity [23]. When the applied load reaches a value of $77 \mathrm{MPa}$ another large strain burst is observed coinciding with the takeoff of the satellite peak (Laue pattern 25). The direction the satellite takes off is situated in between the directions to be expected for slip along $(1 \overline{1} \overline{1})[0 \overline{1} 1]$ and slip along $(\overline{1} \overline{1} 1)[1 \overline{1} 0]$, indicating that at this strain the two other slip systems are already operating. Between the first strain burst and the formation of the satellite peak, the Laue streaking reduces further and the final width of the mother peak is narrower compared to the width prior to loading, demonstrating a partial strain gradient relief in the pillar.

In situ data for the $10 \mu \mathrm{m}$ pillar is shown in section A2.2 of the auxiliary material [22]. The evolution of the vertical axis of the $10 \mu \mathrm{m}$ pillar in the reciprocal space is shown in the inverse pole figure (see Fig. A-3 in [19]) demonstrating that the pillar deforms on the geometrically predicted $(1 \overline{1} \overline{1})[01 \overline{1}]$ slip system resulting in crystal rotation where

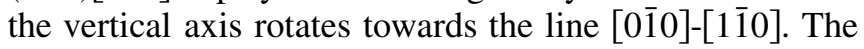
anisotropy of the Laue peak reflections before loading evidence a (11) $[0 \overline{1} \overline{1}]$ strain gradient, however compared to the $2 \mu \mathrm{m}$ pillar the corresponding streaking is far less pronounced. Upon loading, first isotropic peak broadening is observed followed by an increase in the $(1 \overline{1} 1)[0 \overline{1} \overline{1}]$ streaking until an intense subpeak is emitted. The resulting crystal rotation is along the classical geometrically predicted $(1 \overline{1} \overline{1})$ rotation direction. The above observations are confirmed by in situ measurements of two other pillars, namely, a $1.8 \mu \mathrm{m}$ diameter pillar which deforms similarly to the $2 \mu \mathrm{m}$ pillar, initially along a hard slip system, and a $4 \mu \mathrm{m}$ diameter pillar that deforms along the geometrically favorable slip system. Both pillars undergo crystal rotation.

In summary, time-resolved white beam Laue diffraction performed on $2 \mu \mathrm{m}$ diameter Au pillars synthesized using FIB demonstrates that plasticity starts on a hard slip system that is geometrically not predicted, but selected because of the character of the preexisting strain gradient, and that the deformation method involves crystal rotation demonstrating different boundary conditions to what currently is assumed. For the larger Au pillars synthesized by the same method, slip occurs according to classical geometrical predictions involving unconstrained rotation of the crystal with respect to the compression axis. Time-resolved Laue presents a more detailed picture for the "smaller is stronger" paradigm measured on FIB milled metallic pillars and urges to explore the origin of the preexisting strain gradient in terms of FIB damage and the boundary conditions of a microcompression test, providing the correct input for the development of predictive mesoscopic models [24,25].

H. V.S. thanks the Swiss National Science Foundation (No. SNF-2100-065152.01) and the European Commission (No. FP6-NANOMESO) for financial support. The authors also thank Dr. E. Lilleodden for helping out with the calibration of parts of the microcompression and valuable discussions, M. Willimann, A. Bollhalder, and M. Schild for technical support, S. Brandstetter and Dr. B. Schmitt for helping during beam time measurements, and Dr. E. Bitzek for fruitful discussions. Further gratitude is expressed to Dr. U. Hangen and M. Berg from Hysitron Incorporated for supporting the TriboScope $($ implementation.

*Corresponding author.

[1] J. R. Weertman, in Nanostructured Materials: Processing, Properties, and Potential Applications, edited by C.C. Koch (William Andrews, Norwich, NY, 2002), p. 397.

[2] M. A. Meyers, A. Mishra, and D. J. Benson, Prog. Mater. Sci. 51, 427 (2006). 
[3] T. Tabata, H. Fujita, S. Yamamoto, and T. Cyoji, J. Phys. Soc. Jpn. 40, 792 (1976).

[4] S. S. Brenner, J. Appl. Phys. 27, 1484 (1956).

[5] M.D. Uchic, D. M. Dimiduk, J. N. Florando, and W. D. Nix, Science 305, 986 (2004).

[6] C. Motz, T. Schoberl, and R. Pippan, Acta Mater. 53, 4269 (2005).

[7] D. M. Dimiduk, M.D. Uchic, and T.A. Parthasarathy, Acta Mater. 53, 4065 (2005).

[8] M. D. Uchic, D. M. Dimiduk, R. Wheeler, P. Shade, and H. L. Fraser, Scr. Mater. 54, 759 (2006).

[9] J. R. Greer, W. C. Oliver, and W. . Nix, Acta Mater. 53 1821 (2005); 54, 1705 (2006).

[10] J.R. Greer and W.D. Nix, Phys. Rev. B 73, 245410 (2006).

[11] C. A. Volkert and E. T. Lilleodden, Philos. Mag. 86, 5567 (2006).

[12] M. D. Uchic and D. M. Dimiduk, Mater. Sci. Eng. A 400401, 268 (2005).

[13] H. Zhang, B. E. Schuster, Q. Wie, and K. T. Ramesh, Scr. Mater. 54, 181 (2006).

[14] M. D. Uchic and D. M. Dimiduk, Mater. Sci. Eng. A 400401, 268 (2005).

[15] B. Jakobsen, H.F. Poulsen, U. Lienert, J. Almer, S. D. Shastri, H. O. Sørensen, C. Gundlach, and W. Pantleon, Science 312, 889 (2006).

[16] B. C. Larson, W. Yang, G. E. Ice, J. D. Budai, and J.Z. Tischler, Nature (London) 415, 887 (2002).

[17] R. I. Barabash, G. E. Ice, and F. J. Walker, J. Appl. Phys. 93, 1457 (2003).
[18] R. I. Barabash and G. E. Ice, Microdiffraction Analysis of Hierarchical Dislocation Organization, in Encyclopedia of Materials: Science and Technology Updates (Elsevier, Oxford, 2005), p. 1.

[19] See EPAPS Document No. E-PRLTAO-99-002738 for detailed information on the experimental in situ technique. Further results for the discussed smaller $2 \mu \mathrm{m}$ pillar and the summarized results of the larger $10 \mu \mathrm{m}$ pillar are presented. The additional results are supported by movies displaying the Laue peak evolution during compression for both samples. For more information on EPAPS, see http://www.aip.org/pubservs/epaps.html.

[20] R. Maaß, D. Grolimund, S. Van Petegem, M. Willimann, M. Jensen, H. Van Swygenhoven, T. Lehnert, M. A. M. Gijs, C. A. Volkert, E. T. Lilleodden, and R. Schwaiger, Appl. Phys. Lett. 89, 151905 (2006).

[21] L. Margulies, G. Winther, and H. F. Poulsen, Science 291, 2392 (2001).

[22] H. Mughrabi, in Plastic Deformation and Fracture of Materials, edited by R.W. Cahn, P. Haasen, and E. J. Kramer, Materials Science and Technology Vol. 6 (Wiley, Weinheim, 1991).

[23] D. M. Dimiduk, C. Woodward, R. LeSar, and M. D. Uchic, Science 312, 1188 (2006).

[24] V.S. Deshpande, A. Needleman, and E. J. Van der Giessen, J. Mech. Phys. Solids 53, 2661 (2005).

[25] D. Weygand, M. Poignant, P. Gumbsch, and O. Kraft, Mater. Sci. Eng. A (to be published). 